\title{
Possibilities of increase of gyrotron efficiency via frequency locking by external signal
}

\author{
Vladimir L. Bakunin ${ }^{1}$, Gregory G. Denisov ${ }^{1}$, Yury M. Guznov ${ }^{1}$, Michael V. Morozkin ${ }^{1}$, \\ Yulia $V$. Novozhilova ${ }^{1, *}$, and Andrew S. Zuev ${ }^{1}$ \\ ${ }^{1}$ Institute of Applied Physics of Russian Academy of Sciences, Ulianov str., 46, BOX-120, \\ Nizhny Novgorod, Russia
}

\begin{abstract}
Possibility of efficiency increase of a gyrotron, which frequency is locked by an external monochromatic signal, is studied. Dependencies of orbital efficiency on cyclotron frequency resonance mismatch for different currents of the electron beam are found. It is shown that in multi-mode gyrotron there is a possibility of shifting of frequency-locking zone to the region of parameters corresponding to the optimal efficiency, so efficiency may be significantly increased compared to the free gyrotron.
\end{abstract}

\section{Introduction}

During recent years influence of external monochromatic signal on multi-mode gyrotron is studied theoretically [1-3] and experimentally [4]. These studies were initiated by the development of synthesized quasioptical converter in IAP RAS [5], which transforms external signal to the operating mode. The study of frequqency locking in multimode powerful gyrotrons is important for a number of applications, such as spectroscopy, plasma diagnostics and, in prospect, creation of a complex of coherent-radiating gyrotrons, driven by a leading gyrotron with stabilized frequency. The first experimental study of frequency locking of a powerful $35 \mathrm{GHz}$-gyrotron with TE7.3 operating mode by a weak monochromatic signal of magnetron was performed [4]. For a poweful 170-GHz gyrotron with TE28.12 operating mode, the results of our simulations show that in the frequencylocking regime efficiency, maximal achievable currents and bandwidth of frequency alteration can be increased $[1,2]$. However, some questions, concerned to the possibility of efficiency increase of a gyrotron, driven by an external signal, remained insufficiently studied. In this paper, using the two listed gyrotrons (with frequencies of $35 \mathrm{GHz}$ and 170 $\mathrm{GHz}$ ) as an example, we show at what parameters an increase or decrease in efficiency can be expected when the frequency is locked by an external signal.

\section{Calculations of the parameters of gyrotron generation in frequency-locking regime}

Problem of gyrotron frequency locking by external signal was considered within fixedlongitudinal-field structure approximation, which is fulfilled if Q-factor of the resonator is

\footnotetext{
*Corresponding author: julia.novozhilova2009@yandex.ru
} 
quite high. Longitudinal field distribution was set to be the same as for the eigenmode of the cold (without electron beam) gyrotron cavity with one longitudinal variation. Orbital efficiency of the gyrotron - a fraction of transversal energy of the electrons, given to the radiation field and averaged over all the electrons - was determined at the single-mode generation regime from the equation of the electron motion

$$
\frac{d p}{d \varsigma}+i\left(\Delta+|p|^{2}-1\right) p=i F f(\varsigma)
$$

where $p$ - dimensionless transversal momentum of the electrons, entering the interaction space at different phases of the field

$$
p(\varsigma=0)=\exp (i \vartheta), 0 \leq \vartheta<2 \pi,
$$

$\varsigma$ - dimesionless longitudinal coordinate, $F$ and $f(\varsigma)$ - dimensionless amplitude and longitudinal distribution of the field, acting upon the electrons, $\Delta$ - normalized cyclotron resonance mismatch between radiation frequency and gyrofrequency. These parameters are determined in [1,2]. Orbital efficiency depends on the field amplitude, its longitudinal distribution and on cyclotron resonance mismatch $\Delta$.

Field amplitude, in turn, depends on the electron beam current and coupling coefficient between the beam and the wave and on the signal parameters in the case of the influence of external signal.

For qualitative analysis of an external signal influence on the gyrotron efficiency longitudinal field structure may be approximated by a gaussian function $f(\varsigma)=\exp \left(-(2 \varsigma / \mu)^{2}\right)$, though gaussian distribution differs from the eigen-structure, especially on the exit from the resonator of the gyrotron (fig. 1).

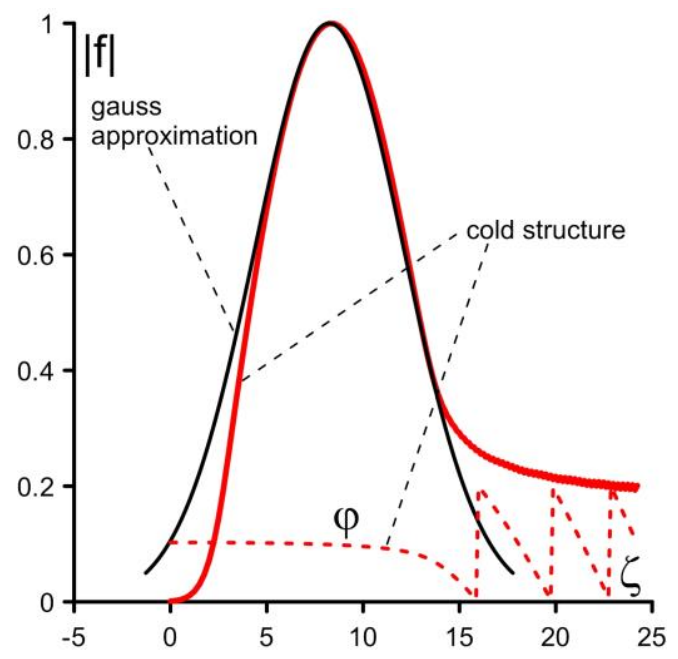

Fig. 1. Longitudinal gaussian distribution of the field and eigen-distribution of the absolute value and phase of the field of the single-humped mode in the cavity of the 35-GHz gyrotron with TE7.3 operating mode.

Approximation of the longitudinal structure of the field by a gaussian curve allows one to use the only parameter for description of this structure - width $\mu$ of the gaussian curve. Fig. 2 represents isolines of orbital efficiency at the plane of two parameters: absolute value of the field amplitude $|F|$ and normalized length of the interaction space $\mu$ for the gaussian longitudinal field structure. Integration of the equation (1) was made in the interval of $\varsigma$ with the bounds, corresponding to the $e^{-3}$-times decrease of the field in 
relation to its maximal value. One can see that if $|F|$ is varying, then efficiency may either increase or decrease, depending on the initial value of the field and normalized length $\mu$.

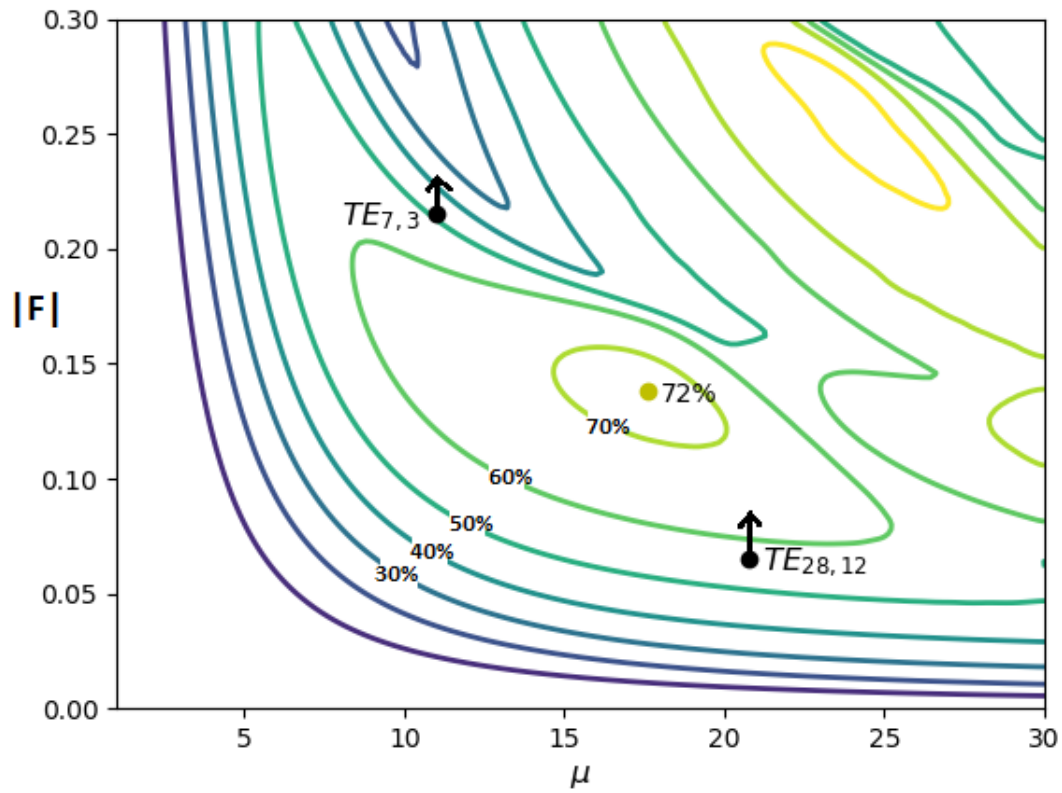

Pic. 2. Lines of equal orbital efficiencies on the plane of normalized field amplitude $|F|$ and normalized length of the interaction space $\mu$. Arrows indicate direction of efficiency variation in the presence of external signal (increase of the field amplitude) for 170-GHz gyrotron (operating mode TE28.12) and 35-GHz gyrotron (operating mode TE7.3).

When gyrotron frequency (as well as frequency of any other autooscillator) is locked by a monochromatic external signal, amplitude of the field and, consequently, the power of the radiation, increases. However, according to the equation of power balance $P_{\text {rad }}=P_{e x}+I U \eta \quad\left(P_{r a d}, P_{e x} \quad-\right.$ powers of radiation and external signal, respectively, $I, U, \eta$ - beam current, voltage and electron efficiency), efficiency can either increase or decrease, because radiation power can either increase due to increase of the electron beam efficiency or due to absorbtion of the power of external signal.

Comparison of the fig. 2 with our calculations of the efficiencies of the $170-\mathrm{GHz}$ and $35-$ $\mathrm{GHz}$ gyrotrons in the frequency locking regime shows their full accordance. In the case of $170-\mathrm{GHz}$ gyrotron, value of the field of the free oscillation is less than optimal, therefore frequency locking and increase of the field amplitude lead to transition to the region of higher efficiencies (fig. 3a, 4a). In the case of 35-GHz gyrotron, value of the field of the free oscillation is higher than optimal, therefore frequency locking and increase of the field amplitude lead to transition to the region of lower efficiencies (fig. 3b, 4b).

\section{Conclusion}

We get full accordance of calculation results for multi-mode gyrotron efficiencies at stationary single-mode frequency locking regime with the results for a more simple models. Based on the calculation performed, one can conclude that efficiency at the frequency locking regime may increase if the field amplitude of the free stationary oscillation (without external signal) is less than the value, optimal for achievement of high efficiency. 


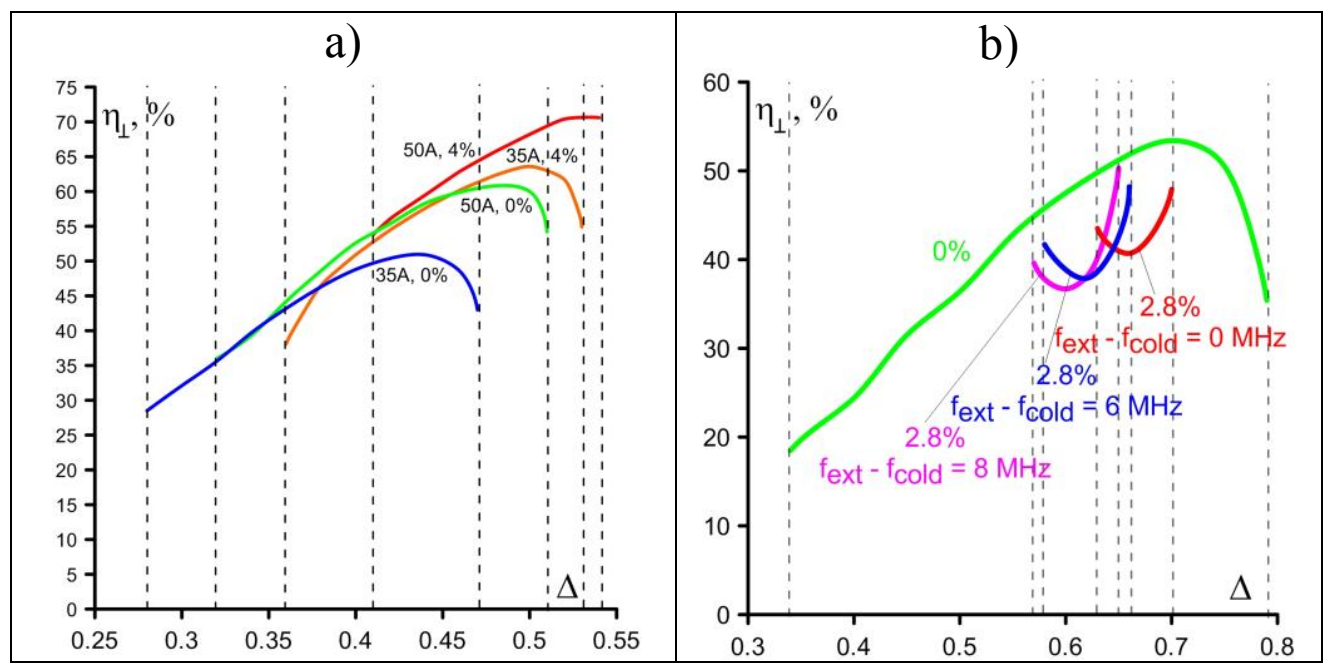

Fig. 3. Dependencies of orbital efficiency on cyclotron resonance mismatch for the 170-GHz gyrotron (a) and 35-GHz gyrotron (b). Different curves correspond to different powers of external signal (in percents of the power of radiation of the free gyrotron), different values of the beam current (fig. 3a) and different frequencies of external signal (fig. 3b).

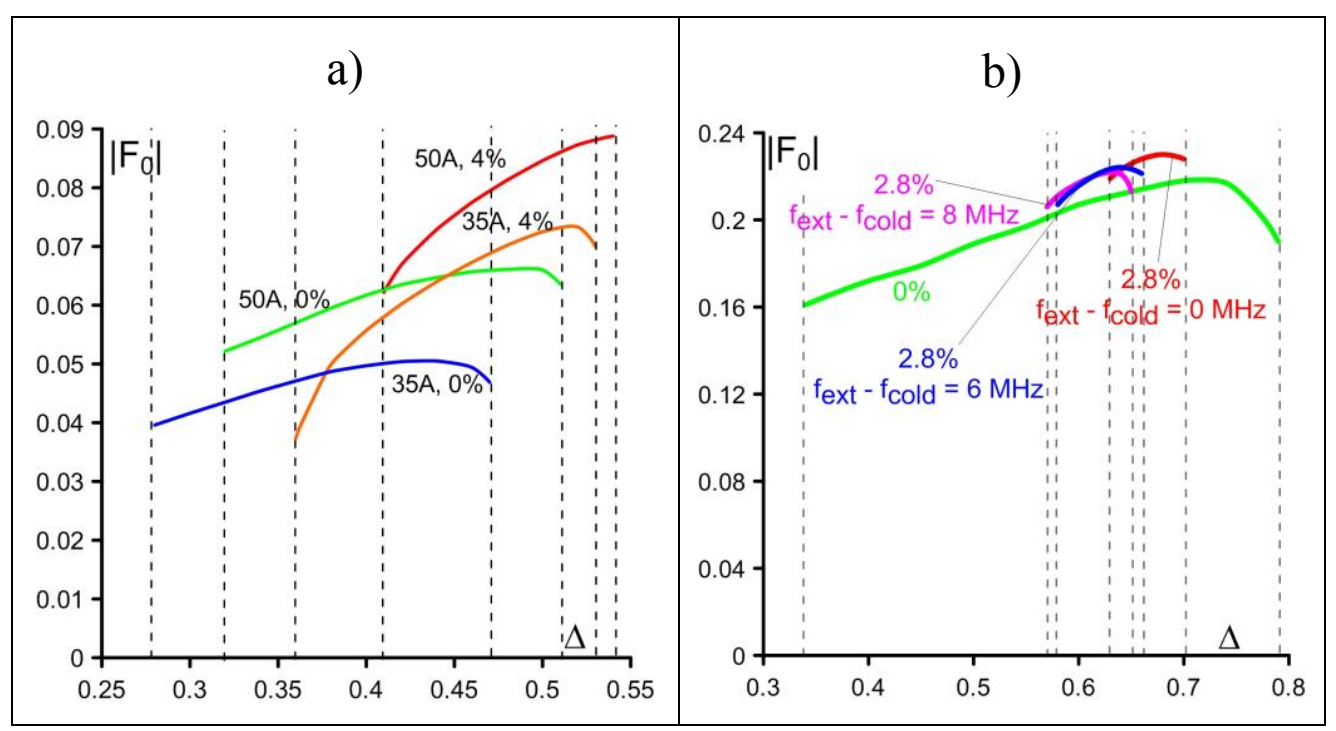

Fig. 4. Dependencies of the field amplitude on cyclotron resonance mismatch for the $170-\mathrm{GHz}$ gyrotron (a) and 35-GHz gyrotron (b). Different curves correspond to different powers of external signal (in percents of the power of radiation of autonomic gyrotron), different values of the beam current (fig. 4a) and different frequencies of external signal (fig. 4b).

The work was supported by Russian Fund of Basic Research, grants \# 18-02-00839 and 17-02-00183.

\section{References}

1. V.L. Bakunin, G.G. Denisov, Yu.V. Novozhilova, A.P. Fokin. Mode Competition Effect on Frequency Locking of a Multimode Gyrotron by a Monochromatic External Signal. Radiophysics and Quantum Electronics, Volume 59, Issue 8-9, pp. 638-647. (January 2017) 
2. V.L. Bakunin, G.G. Denisov, Yu.V. Novozhilova. Zones of Frequency Locking by an External Signal in a Multimode Gyrotron of a Megawatt Power Level. Radiophysics and Quantum Electronics. Volume 58, Issue 12, PP 893-904 (May 2016)

3. N.S. Ginzburg, A.S. Sergeev, I.V. Zotova. Time-domain self-consistent theory of frequency-locking regimes in gyrotrons with low- $Q$ resonators. Physics of Plasmas. V. 22. P. 033101-1-5 (2015)

4. V.L. Bakunin, Yu.A. Guznov, G.G. Denisov, N.I. Zaitsev, S.A. Zapevalov, A.N. Kuftin, Yu.V. Novozhilova, A.P. Fokin, A.V. Chirkov, A.S. Shevchenko. An Experimental Study of the Influence of an External Signal on the Generation Mode of a Megawatt-Power Gyrotron. Technical Physics Letters. Volume 44, Issue 6, PP 473475 (June 2018)

5. A.V. Chirkov, G.G. Denisov, A.N. Kuftin. Perspective gyrotron with mode converter for co- and counter-rotation operating modes. Applied Physics Letters 106, 263501 doi: 10.1063/1.4923269 (2015)

6. G.S. Nusinovich, R.E. Erm. Efficiency of the CRM-monotron with a Gaussian axial distribution of the high-frequency field. Elektronnaya Tekhnika, Ser.1. Elektronika SVCh. No. 8, pp 55-60 (in Russian) (1972) 